\title{
Cognitive Time Scales in a Necker-Zeno Model for Bistable Perception
}

\section{H. Atmanspacher ${ }^{1,2},{ }^{*}$ M. Bach $^{3}$, T. Filk ${ }^{1,2,4}$, J. Kornmeier ${ }^{1,3}$ and H. Römer ${ }^{4}$}

1 - Institute for Frontier Areas of Psychology and Mental Health, Wilhelmstr. 3a, 79098 Freiburg, Germany

2 - Parmenides Center, Via Mellini 26-28, 57031 Capoliveri, Italy

3 - Department of Ophtalmology, University of Freiburg, Killianstr. 5, 79106 Freiburg, Germany

4 - Institute of Physics, University of Freiburg, Hermann-Herder-Str. 3, 79104 Freiburg, Germany

\begin{abstract}
The "Necker-Zeno model", a model for bistable perception inspired by the quantum Zeno effect, was previously used to relate three basic time scales of cognitive relevance to one another in a quantitative manner. In this paper, the model predictions are compared with experimental results obtained under discontinuous presentation of an ambiguous stimulus. In addition to earlier results for long inter-stimulus intervals, we show that the reversal dynamics according to the Necker-Zeno model is also in agreement with new results for short inter-stimulus intervals. Moreover, we refine the model in such a way that it accounts for the distribution of "dwell times" (inverse reversal rates). Finally, we indicate applications concerning the modification of cognitive time scales under conditions of psychopathological impairments and meditationinduced modes of awareness.
\end{abstract}

*Address correspondence to this author at the Theory Division of the Institute for Frontier Areas of Psychology, Wilhelmstr. 3a, D-79098 Freiburg, Germany; E-mail: haa@igpp.de 


\section{Introduction}

The bistable perception of ambiguous stimuli or in binocular rivalry is well established as an interesting arena to study phenomena of attention and perceptual awareness and their neural basis [1-4]. Binocular rivalry and perceptual reversals of ambiguous stimuli have several features in common. Examples are: the form of the distribution of dwell times (inverse reversal rates) for a given stimulus [5,6], a high inter-individual variability in reversal rates [5,7], and a sizable influence of physical stimulus properties $[8,9]$.

But there are also indications for differences between ambiguous stimuli and binocular rivalry. For instance, voluntary control of subjects over reversal rates seems to be pronounced for ambiguous stimuli [10] but quite limited for binocular rivalry $[11,12]$. This has been interpreted in terms of a higher influence of bottom-up, stimulus-driven processing for binocular rivalry as compared to ambiguous stimuli. A good review of the current state of knowledge on binocular rivalry is due to Alais and Blake [13].

A number of EEG studies of perceptual reversals have found ERP (event-related potential) correlates. Basar-Eroglu et al. [14] reported a P300-like component with perceptual reversals, which was interpreted as a correlate of cognitive processing. Kornmeier and Bach [15] were able to detect earlier ERP components starting at 130 msec with a refined experimental design. In the frequency domain, both increased $\gamma$-activity [16] and decreased $\alpha$-activity [17] were reported to occur in the time range of the P300 component.

In this contribution, we consider bistable perception from a theoretical perspective. Different from numerous theoretical approaches in the literature (e.g., [18-20], and others) we do not primarily look for neural mechanisms implementing the reversal dynamics. Our model (first proposed in [21]) has been worked out in the spirit of a system-theoretical framework that can be applied to cognitive states and properties without requiring an explicit discussion of their neural correlates. (Of course, such correlates will be a desirable further ingredient of the model if they can be determined.) The model relates the dwell time in bistable perception, which has been theoretically studied extensively (see, e.g., [6,22-25]), to other important cognitive time scales.

The proposed model is called the Necker-Zeno model and will be shortly reviewed in Sec. 2. In Sec. 3 we implement a refinement of the model that allows us to describe the distribution of dwell times. In Sec. 4 we show that a predicted quantitative relationship between different cognitive time scales, which was earlier confirmed by data from [26], is also in agreement with new experimental material from [27]. In Sec. 5 we predict that particular cognitive time scales should be dramatically changed during particular modes of awareness that are restricted or enhanced with respect to "normal" cognition. Future experimental work to test corresponding conjectures is proposed. 


\section{Necker-Zeno Model for Bistable Perception}

A theoretical approach to describe the dynamics of alternating perceptive configurations was recently proposed in terms of the so-called Necker-Zeno model [21]. This model is inspired by the Zeno effect for unstable quantum states [28] and describes the perceptual instability of ambiguous stimuli in a formal fashion. In contrast to attempts to apply standard quantum physics to brain functioning and consciousness directly, the Necker-Zeno model is based on a generalized formal framework, particularly suited for applications beyond physics [29]. Earlier suggestions to use Zeno-type arguments for cognitive systems are due to [30,31].

A key assumption of the Necker-Zeno model is that the cognitive state corresponding to a perceived stimulus is updated at intervals $\Delta T$ (of the order of $30 \mathrm{msec}$ to $70 \mathrm{msec}$, see below). The probability that no reversal occurs within a duration $T$ between two successive updates is then given by:

$$
w(T)=\cos ^{2}(g T) \quad \text { with } g=\frac{\pi}{4 t_{0}},
$$

where $t_{0}$ characterizes the period of the reversal dynamics assuming no updates (of the order of $300 \mathrm{msec}$, see below). The inverse of $t_{0}, g$, determines how fast the cognitive state corresponding to a perceived stimulus decays.

Let $\left\{\tau_{i}\right\}_{i=0, \ldots, N}$ be the instants at which an update of the cognitive state has been performed, and let $w\left(\tau_{N}, \tau_{N-1}, \ldots, \tau_{1}, \tau_{0}=0\right)$ be the joint probability that no perceptual reversal has occured from $\tau_{0}$ up to $\tau_{N}=T$. Then

$$
W(T):=w\left(\tau_{N}, \tau_{N-1}, \ldots, \tau_{1}\right)=\prod_{i=1}^{N} \cos ^{2}\left(g\left(\tau_{i}-\tau_{i-1}\right)\right)=\prod_{i=1}^{N} \cos ^{2}(g \Delta T(i)),
$$

with

$$
\Delta T(i)=\tau_{i}-\tau_{i-1}
$$

For the Necker-Zeno model we have $\Delta T(i) \ll t_{0}$, so we may expand the cosine up to the quadratic term:

$$
W(T) \approx \prod_{i=1}^{N} \mathrm{e}^{2 \ln \left(1-\frac{1}{2} g^{2}\left(\Delta T_{i}\right)^{2}\right)} \approx \mathrm{e}^{-g^{2} \sum_{i=1}^{N}\left(\Delta T_{i}\right)^{2}} .
$$

Assuming a constant updating interval $\Delta T(i)=\Delta T$, we obtain

$$
W(T)=\mathrm{e}^{-g^{2} N(\Delta T)^{2}},
$$

which means for $T=N \Delta T$ :

$$
W(T)=\mathrm{e}^{-g^{2} \Delta T \cdot T} .
$$

$W(T)$ is the probability that no reversal has occurred up to time $T$. Hence, $1-W(T)$ describes the integrated (cumulative) distribution of "dwell times" (inverse 
reversal rates). It yields the following probability distribution (density) for dwell times:

$$
P(T)=-\frac{\mathrm{d} W(T)}{\mathrm{d} T}=\gamma \mathrm{e}^{-\gamma T},
$$

where $\gamma=g^{2} \Delta T$. The mean dwell time $\langle T\rangle$ is given by:

$$
\langle T\rangle=\frac{1}{\gamma}=\left(\frac{16}{\pi^{2}}\right) \frac{t_{0}^{2}}{\Delta T},
$$

leading to the relation

$$
\Delta T \cdot\langle T\rangle=C t_{0}^{2},
$$

where $C$ is of the order of 1 such that $t_{0}$ is basically the geometric mean of $\langle T\rangle$ and $\Delta T$.

In this way, the Necker-Zeno model predicts a quantitative relationship between three time scales which can be interpreted in terms of cognitive time scales (for more details see [21]):

(i) The time between successive information updates of the cognitive state is related to the so-called sequential order threshold of $\Delta T \approx 30 \mathrm{msec}$ [32]. In the original quantum Zeno effect $\Delta T$ is the time between successive observations.

(ii) The decay time for a sensory input to become consciously accessible (cognitively processed) is of the order of $t_{0} \approx 300 \mathrm{msec}$ [14]. In the original quantum Zeno effect $t_{0}$ is the oscillation period between the two unstable states without updates, a situation which is of more or less hypothetical character in cognition.

(iii) The observed mean dwell time $\langle T\rangle$ between successive reversals of competing configurations of an ambiguous stimulus is usually of the order of $3 \mathrm{sec}$ [32].

These cognitive time scales obviously satisfy Eq. (5). More detailed empirical tests of Eq. (5) are possible if one of the time scales can be measured as a function of another one, which is experimentally controllable, while the third one is considered fixed. We come back to this option in Sec. 4 .

\section{Distribution of Dwell Times}

The Necker-Zeno model as introduced in the preceding section predicts an exponential decrease for $W(T)$ according to Eq. (2) and, hence, a Poisson distribution for $P(T)$ according to Eq. (3). Early observations [5] revealed, however, that the dwell time distribution resembles a gamma distribution of the form

$$
P(T) \propto T^{b} \mathrm{e}^{-\gamma T}
$$

In order to reproduce this observed behavior of the dwell time distribution, we may refine the Necker-Zeno model with $b=0$ (cf. Eq. (3)) in one of two possible ways. We can assume that either the decay parameter $g$ or the updating interval $\Delta T$ is non-constant during an initial (transient) phase of the process. Indications for such 
an initial phase were earlier reported $[33,34]$ with increased dwell times during the initial stage of stimulus observation.

\subsection{Non-Constant Decay $g$}

Suppose that the parameter $g$ depends on the number of previous updates. We set

$$
g(i)=g \cdot f(i),
$$

where $f(i)$ is a function which starts from 0 and approaches 1 for large $i$. Now we consider $f$ to be of the form

$$
f(i)=\tanh \left(\frac{i}{\alpha}\right),
$$

where $\alpha$ determines the number of updates $N_{c}$ within the transient period during which $g(i)$ increases up to its asymptotic value. As $f(i)$ grows approximately linearly for small values of $i$, we determine $\alpha$ from the condition that the argument of the tanh is one:

$$
\frac{N_{c}}{\alpha}=1 \text {. }
$$

The relation between (ordinary) time $T$ and the number of updates $N$ is linear,

$$
T=T(N)=N \Delta T,
$$

and leads to the condition:

$$
\alpha=\frac{T_{c}}{\Delta T} .
$$

$T_{c}$ is the time after which $f(i)$ has roughly approached its asymptotic value.

\subsection{Non-Constant Updating $\Delta T$}

As an alternative to an initial increase of $g$ (Sec. 3.1), we now leave $g$ timeindependent and suppose that the updating interval $\Delta T$ is not constant:

$$
\Delta T(i)=\tau_{i}-\tau_{i-1}=\Delta T \cdot f(i),
$$

where $f(i)$ is defined as above. Again we posit the condition

$$
\frac{N_{c}}{\alpha}=1,
$$

but now the relation between the number $N$ of updates and time $T$ is more complicated:

$$
T=T(N)=\sum_{i=1}^{N} \Delta T(i)=\Delta T \sum_{i=1}^{N} f(i) .
$$


For the determination of $\alpha$ we take $f(i)$ to be approximately linear for $i<N_{c}$ and obtain an approximate relation between $N_{c}$ and $T_{c}$, now understood as the time after which $\Delta T$ has approached its asymptotic value:

$$
\Delta T \sum_{i=1}^{N_{c}}\left(\frac{i}{\alpha}\right)=\Delta T \frac{N_{c}\left(N_{c}-1\right)}{2 \alpha}=T_{c} .
$$

From this follows for $\alpha=N_{c}$ (the same condition as in Sec. 3.1):

$$
\frac{(\alpha-1)}{2}=\frac{T_{c}}{\Delta T}
$$

or, roughly,

$$
\alpha=\frac{2 T_{c}}{\Delta T} .
$$

This result differs from the result for constant $\Delta T$ by a factor of 2 .

\subsection{Comparison}

We now compare the behavior of the dwell time distribution for the two cases described in Secs. 3.1 and 3.2. In both cases, we obtain for the integrated dwell time distribution introduced in Sec. 2:

$$
w\left(\tau_{N}, \tau_{N-1}, \ldots, 1\right)=\prod_{i=1}^{N} \cos ^{2}(g f(i) \Delta T) \approx \mathrm{e}^{-g^{2}(\Delta T)^{2} \sum_{i} f(i)^{2}},
$$

with $\tau_{i}=\Delta T \sum_{j=1}^{i} f(j)$. So we find

$$
W(T)=\exp \left(-g^{2}(\Delta T)^{2} \sum_{i=1}^{N} f(i)^{2}\right),
$$

where $T$ as a function of the number $N$ of updates is given by

$$
\begin{aligned}
& T(N)=\Delta T \cdot N \quad \text { for non-constant } g, \\
& T(N)=\Delta T \sum_{i=1}^{N} f(i) \quad \text { for non-constant } \Delta T \text {. }
\end{aligned}
$$

Strictly speaking, these relations have to be solved for $N$ in order to obtain $N(T)$ which then yields $W(T)=W(N(T))$.

Approximating sums by integrals, we have:

$$
W(T)=\exp \left(-g^{2}(\Delta T)^{2} \int_{0}^{N} f(x)^{2} \mathrm{~d} x\right)
$$


with

$$
\begin{aligned}
& T(N)=\Delta T \cdot N \quad \text { for non-constant } g, \\
& T(N)=\Delta T \int_{0}^{N} f(x) \mathrm{d} x \quad \text { for non-constant } \Delta T .
\end{aligned}
$$

$P(T)$ is obtained by taking the derivative with respect to $T$ (cf. Eq. (3)):

$$
P(T)=-\frac{\mathrm{d} W(T)}{\mathrm{d} T}=-\frac{\mathrm{d} W(N)}{\mathrm{d} N} \cdot \frac{\mathrm{d} N}{\mathrm{~d} T} .
$$

While the first factor in $P(T)$,

$$
\frac{\mathrm{d} W(N)}{\mathrm{d} N}=-g^{2}(\Delta T)^{2} f(T)^{2} \exp \left(-g^{2}(\Delta T)^{2} \int_{0}^{N} f(x)^{2} \mathrm{~d} x\right),
$$

is the same in both cases, we find for the inverse of the second factor in $P(T)$ :

$$
\frac{\mathrm{d} T(N)}{\mathrm{d} N}=\Delta T \quad \text { for non-constant } g,
$$

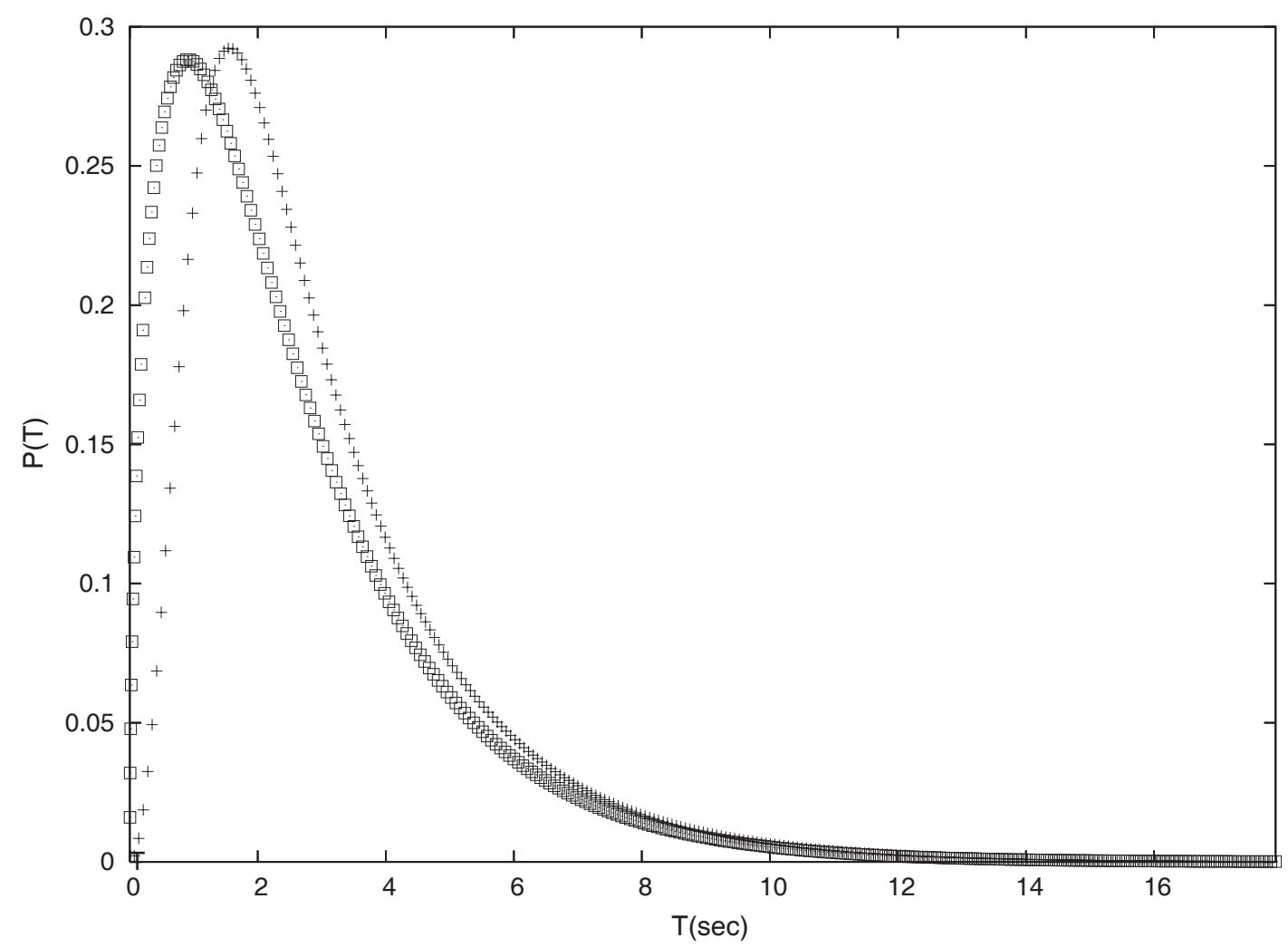

Figure 1: Distribution $P(T)$ according to Eqs. $(8,9)$, where the initial behavior of the reversal dynamics is implemented (a) due to an increasing update interval $\Delta T$ (squares) and, alternatively, (b) due to an increasing decay parameter $g$ (crosses). Parameters are $t_{0}=300 \mathrm{msec}$ and $\Delta T=70 \mathrm{msec}$ (reached after about one second in case (a)). 


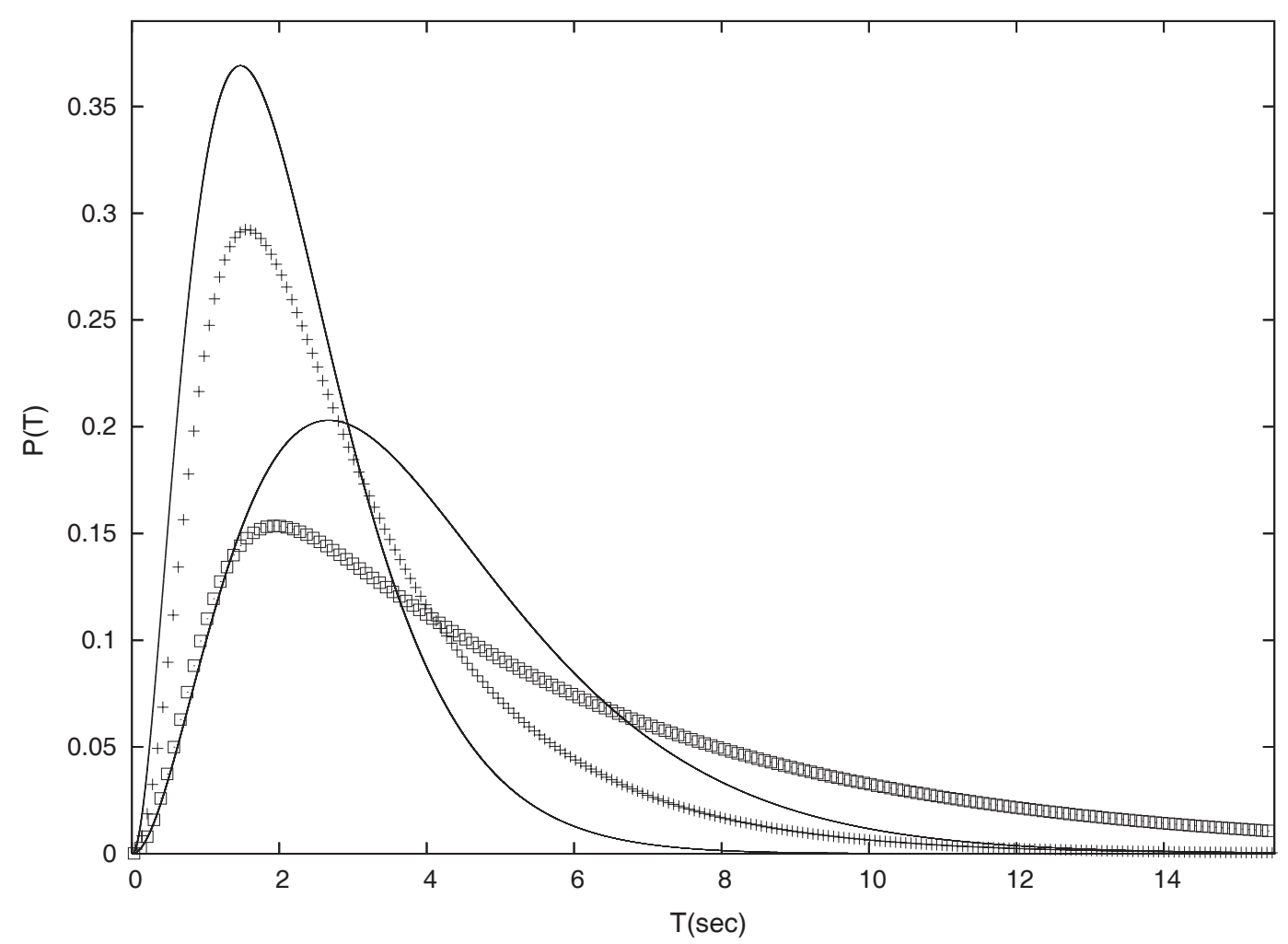

Figure 2: Distribution $P(T)$ for $t_{0}=300 \mathrm{msec}$ according to (a) gamma distributions (solid lines) with $b=2$ for $\Delta T=70 \mathrm{msec}$ (highest maximum) and $\Delta T=30 \mathrm{msec}$; (b) according to Eq. (8) for non-constant $g$, and with $\Delta T=70$ msec (crosses) and $\Delta T=30$ msec (squares). Gamma distributions decay considerably faster for larger $T$.

$$
\frac{\mathrm{d} T(N)}{\mathrm{d} N}=\Delta T f(T) \quad \text { for non-constant } \Delta T .
$$

So we obtain:

$$
\begin{aligned}
& P(T)=g^{2}(\Delta T) f(T)^{2} \exp \left(-g^{2}(\Delta T)^{2} \int_{0}^{N} f(x)^{2} \mathrm{~d} x\right) \text { for non-constant } g \\
& P(T)=g^{2}(\Delta T) f(T) \exp \left(-g^{2}(\Delta T)^{2} \int_{0}^{N} f(x)^{2} \mathrm{~d} x\right) \text { for non-constant } \Delta T .(9)
\end{aligned}
$$

One difference between these two expressions, which hold for any monotonic function $f(T)$, derives from the fact that time $T$ as a function of $N$ has to be obtained according to different prescriptions (see Eq. (7)). But the main difference is that for non-constant $g, P(T)$ is proportional to $f(T)^{2}$, while for non-constant $\Delta T$ it is proportional to $f(T)$. Depending on the power-law behavior of $f(T)$ as a function of small $T$, this leads to a different behavior in $P(T)$.

In Figure 1, the two distributions according to Eqs. $(8,9)$ for $\Delta T=70 \mathrm{msec}$, $t_{0}=300 \mathrm{msec}$, and $T_{c}=1.05 \mathrm{sec}$ are compared. The figure shows that the increase 
of $P(T)$ for small $T$ is steeper for non-constant $\Delta T$. Already the early observations of dwell times by Borsellino et al. [5] (similarly [24]) indicate that $P(T)$ follows a high power of $T$ for small $T$, and that $\langle T\rangle$ is approximately twice as large as the standard deviation of $P(T)$.

In principle, good fits to experimental dwell times can be obtained for both Eq. (8) and (9), depending on the choice of $f(T)$. However, assuming an intuitively plausible low power for $f(T)$, for instance $\tanh (T)$, suggests that the slower increase of $P(T)$ due to a non-constant $g$ fits the experimentally obtained results better. (The mentioned observations by Price [34] also support an initial variability of $g$ rather than $\Delta T$.) Moreover, the ratio of $\langle T\rangle$ and the standard deviation of $P(T)$ in Figure 1 is closer to the experimentally found result of 2 for non-constant $g$. Therefore, Figure 2 shows a comparison of gamma distributions with distributions according to Eq. (8) (time-dependent $g$ ) for $\Delta T=70 \mathrm{msec}$ and $\Delta T=30 \mathrm{msec}$.

It is tempting to speculate about a cognitive interpretation of the time-dependence of $g$ or $\Delta T$. In a vague sense, which needs to be made more precise, one might argue that an initially focused and subsequently decreasing attention could be an interesting candidate. This would mean that increased attention can both accelerate the updating of a given cognitive state and decelerate the decay out of this state. Our model favors the second option, but in order to confirm that this has in fact to do with attention, additional independent pieces of evidence would be necessary.

For instance, one might speculate that recent evidence $[11,12]$ for voluntary control over dwell times in the perception of ambiguous figures - as opposed to binocular rivalry - would imply a significant contribution of top-down processing - as opposed to bottom-up processing. The times scales involved should thus be longer for bistability in ambiguous perception. Moreover, it was found that dwell times increase if attention is distracted [35] and that dwell times are reduced by increasing vigilance [36]. Further experimental investigations of the relation between volitional attention or vigilance and dwell times are in progress.

\section{Cognitive Time Scales under Discontinuous Stimulus Presentation}

Research on perceptual reversals of ambiguous stimuli started with continuous stimulus presentation, but Orbach et al. $[37,38]$ proposed discontinuous presentation as an important alternative as early as in the 1960s. In this presentation mode, the stimulus is interrupted by inter-stimulus intervals (or off-times) $t_{\text {off }}$. Variation of $t_{\text {off }}$ was shown to have a significant influence on dwell times $\langle T\rangle$. Their results were later reproduced [26,27,39-41]. Smallest dwell times $\langle T\rangle$ (highest reversal rates) occur for $t_{\text {off }}$ of the order of $t_{0} \approx 300$ msec. For $t_{\text {off }} \leq t_{0},\langle T\rangle$ increases to the value observed under continuous presentation $[27,37,38]$, and for $t_{\text {off }} \geq t_{0},\langle T\rangle$ can increase as much as providing a reversal rate of close to zero $[39,40]$. 


\section{1. $t_{\text {off }} \geq 300 \mathrm{msec}$}

In the experiments presented in [26], the stimulus was a Necker lattice, consisting of 3 by 3 Necker cubes. The stimuli were presented to 10 subjects on a computer screen with on-and off-times of 50, 100, 400, 700, and 1000 msec. For each block of 60 seconds, another combination of $t_{\text {on }}$ and $t_{\text {off }}$ was chosen. Between blocks, a blank screen was shown for 30 seconds. Subjects indicated a perceptual reversal by pressing a button.

In agreement with earlier observations, it was found [26] that $\langle T\rangle$ is more or less independent of $t_{\text {on }}$. The significant dependence of $\langle T\rangle$ on $t_{\text {off }}$, on the other hand, can be compared with the prediction of the Necker-Zeno model. In this model, it can be argued that the off-times $t_{\text {off }}$ in discontinuous presentation represent a "forced" decay time $t_{0}$ as long as $t_{\text {off }} \geq 300 \mathrm{msec}$ (for details see [21]).

Under this assumption, the Necker-Zeno model has been experimentally confirmed with data from [26,38]. Figure 3 (reproduced from [21]) shows mean dwell times $\langle T\rangle$ as a quadratic function of $t_{\text {off }} \approx t_{0}$ as predicted by Eq. (5). In addition to and independent of the quadratic dependence that the model predicts, the best polynomial fit to the data is also quadratic and yields $\Delta T \approx 70 \mathrm{msec}$. (We found that linear and cubic fits have a variance by a factor of 2.7 and 3.7 higher than the variance of the quadratic fit.)

Note that the lowest off-time $t_{\text {off }}=200 \mathrm{msec}$ in Figure 3 has the largest relative deviation from the predicted curve. In the subsequent subsection we will show that this is consistent with our model for off-times $t_{\text {off }} \leq 300 \mathrm{msec}$, where $\langle T\rangle$ changes its functional dependence on $t_{\text {off }}$ qualitatively and increases with decreasing $t_{\text {off }}$.

\section{2. $t_{\text {off }} \leq 300 \mathrm{msec}$}

For off-times smaller than $300 \mathrm{msec}, t_{\text {off }}$ can no longer be used to mimic $t_{0}$ but still has an influence on $\langle T\rangle$. In order to investigate this influence, we analyzed data from [27] in which the Necker lattice was presented to 12 subjects. The presentation time was constant at $800 \mathrm{msec} \pm$ a randomly varied addition between 0 and $100 \mathrm{msec}$ (see below), and $t_{\text {off }}$ was randomly chosen among 14, 43, 130, and $390 \mathrm{msec}$, where the last value served to identify the transition to long inter-stimulus intervals. All off-times occurred equally often. The randomized sequence of off-times avoided habituation effects due to identical successive off-times. Subjects indicated a perceptual reversal by pressing a button. If a reversal was reported, the subsequent off-time was set to $1000 \mathrm{msec}$, and the observation sequence was restarted.

Since the off-times were varied randomly over time, it is not appropriate to consider reversal rates relative to observation time. Instead, the number of reversals following each off-time was counted and related to the total number of occurrences of the corresponding off-time. The dependence of $\langle T\rangle$ on $t_{\text {off }}$ for short off-times as observed in $[37,38]$ was essentially reproduced by the results of [27]. We now show that, in addition to describing the behavior of dwell times for long off-times, the 


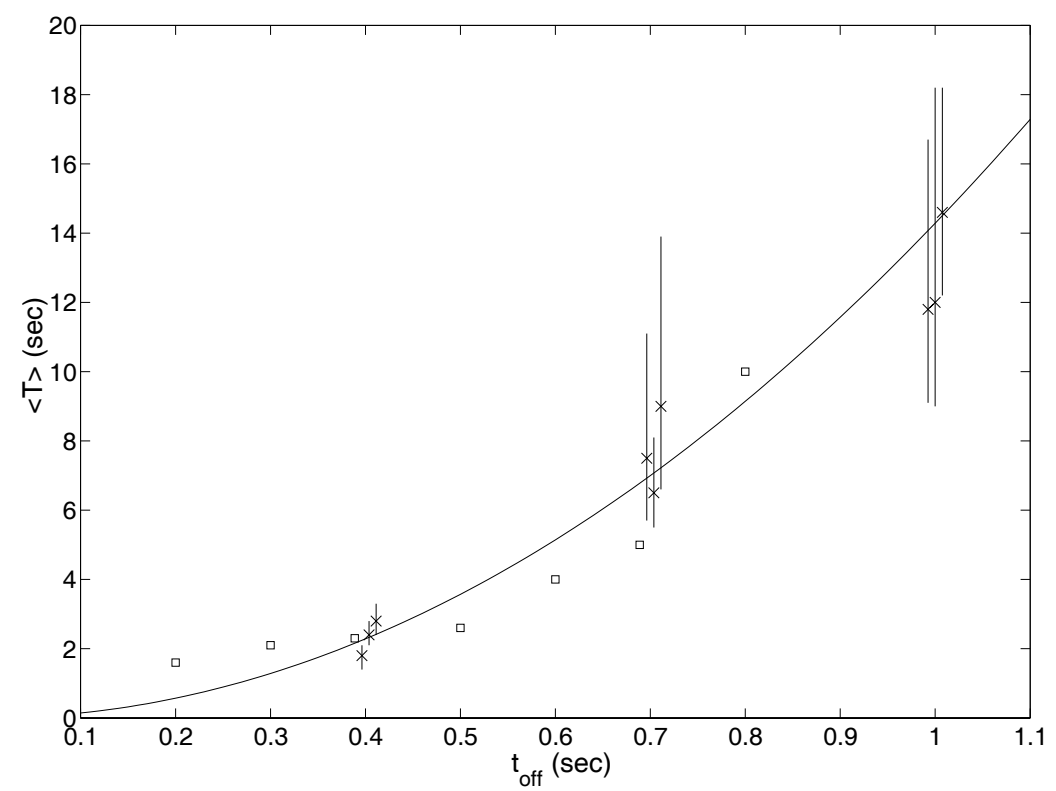

Figure 3: Experimentally obtained mean dwell times $\langle T\rangle$ (inverse reversal rates) for the bistable perception of a discontinuously presented Necker cube. Crosses mark results from [15]; for each off-time, $\langle T\rangle$ (including standard errors) is plotted for three on-times of 0.05 sec, $0.1 \mathrm{sec}$, and $0.4 \mathrm{sec}$. Squares mark results from [38] for an on-time of $0.3 \mathrm{sec}$ (no errors indicated in [38]). The plotted curve shows $\langle T\rangle$ as a function of off-times $t_{\text {off }}$ according to Eq. (5) with $\Delta T \approx 70$ msec. (Reproduced from [21] with permission, (C) Springer-Verlag, Heidelberg.)

Necker-Zeno model is also capable of describing reversal rates as a function of small off-times.

First, we calculated $W(T)$ according to Eq. (6) with $T=\Delta T \cdot N$ (the case where $g$ is time-dependent) for $f(T)=\tanh (T)$ and for on-times $T_{n}^{\text {on }}=(700+n 100 / 7) \mathrm{msec}$ $(n=0, \ldots, 14) . \quad(100 / 7 \approx 14$ msec corresponds to the duration of one image on a screen operating with a frequency of $70 \mathrm{~Hz}$.) This corresponds to the on-times chosen in [27] of $800 \mathrm{msec} \pm$ a randomly chosen time-interval between 0 and $100 \mathrm{msec}$.

Second, we multiplied the averaged on-time probability with $w_{\text {off }}(T)$ according to Eq. (1) for off-times $T_{k}^{\text {off }}=k 100 / 7 \mathrm{msec}$ for the values $k=1,3,9$. This corresponds to Kornmeier et al.'s (2007) choices of $T_{k}^{\text {off }}=14,43,130 \mathrm{msec}$. $T_{k}^{\text {off }}=390 \mathrm{msec}$ is outside the scope of small off-times. The mean dwell time of $2.7 \mathrm{sec}$, corresponding to the reversal rate of $0.36 / \mathrm{sec}$ shown in Figure 4, is in excellent agreement with the observations for large off-times in Figure 3. Note that Eq. (5) remains valid whenever $\langle T\rangle \gg t_{0}$. The increase of $\langle T\rangle$ for decreasing $t_{\text {off }}<300 \mathrm{msec}$ is due to the fact that in this regime $t_{0}$ is not approximated by $t_{\text {off }}$.

The results for small off-times are the three probabilities

$$
w_{k}=\overline{W(T=800 \pm n 100 / 7)} w_{\text {off }}\left(T_{k}\right),
$$




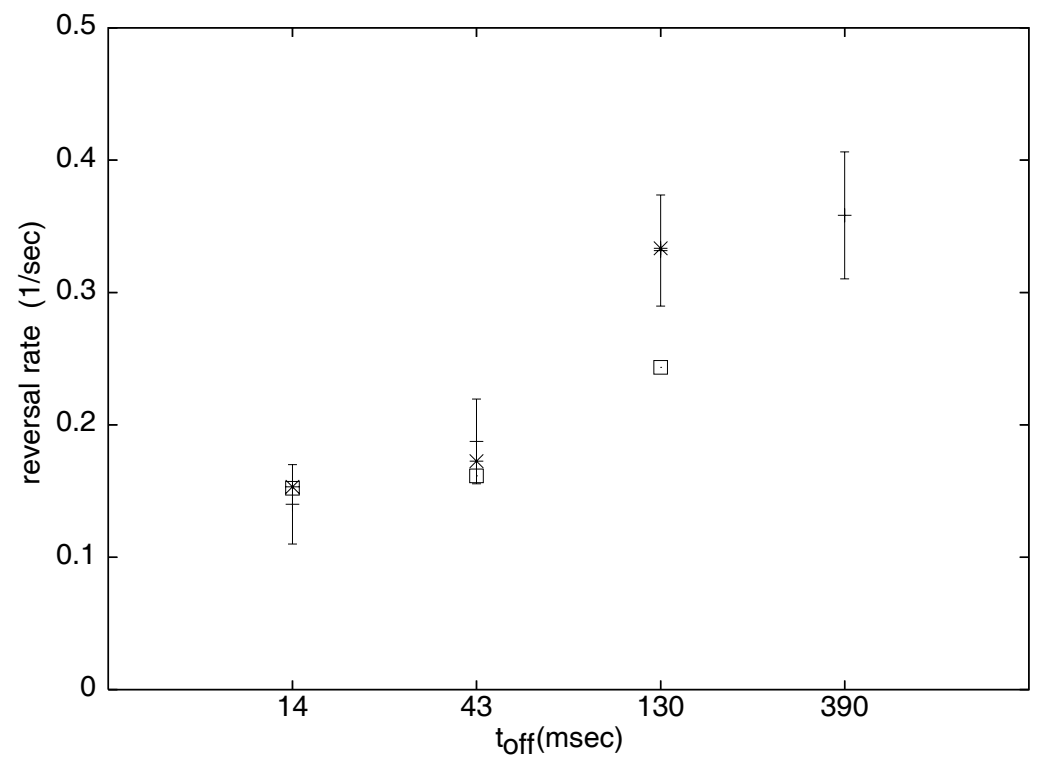

Figure 4: Reversal rates versus off-times $t_{\text {off }}$ : (a) Experimental observations from [27], with standard error of the mean for the corresponding population; (b) best fit of $\left(1-w_{k}\right)$ to those experimental data according to the Necker-Zeno model (asterisks), yielding $\Delta T \approx 16$ msec, $t_{0} \approx 210 \mathrm{msec}$, and $T_{c} \approx 63 \mathrm{msec} ;$ (c) results (squares) for assumed parameters $\Delta T=30 \mathrm{msec}$ and $t_{0}=300 \mathrm{msec}$. Values for (b) are calculated under the condition of an initially increasing $g$. The reversal rate for $t_{\mathrm{off}}=390 \mathrm{msec}$ is disregarded in the fit since it is outside the scope of small off-times.

where $\overline{W(T)}$ is a mean value over $n=0, \ldots, 14$. The probabilities $\left(1-w_{k}\right)$ represent reversal rates and are plotted in Figure 4 for two different sets of parameters $\Delta T$, $t_{0}$ and $T_{c}$.

- Asterisks in Figure 4 show reversal rates $w_{k}$ for $\Delta T=16 \mathrm{msec}, T_{c}=63 \mathrm{msec}$, and $t_{0}=209.4 \mathrm{msec}$, obtained from a least-squares fit of the reversal rates according to the Necker-Zeno model with the reversal rates observed in [27]. Note, however, that we cannot determine $\Delta T$ and $T_{c}$ separately, because they appear in combined form in $\overline{W(T)}$. Table 1 shows combinations of $\Delta T$ and $T_{c}$ within a range of $0.025 \%$ of the variance of the best fit providing $t_{0}=209.4$ msec. All combinations listed in Table $\mathbf{1}$ are consistent with the measured dwell times.

- Squares in Figure 4 show reversal rates $w_{k}$ for $\Delta T=30 \mathrm{msec}, t_{0}=300 \mathrm{msec}$ and $T_{c}=0$, the parameters of the original Necker-Zeno model reviewed in Sec. 2. The deviating reversal rate for $t_{\text {off }}=130$ msec suggests that slightly smaller values of $t_{0}$ and/or $\Delta T$ might be more appropriate. 


$\begin{array}{cccccccccccccccc}\Delta T & 14.5 & 16 & 18 & 20 & 22 & 26.5 & 37 & 38 & 41.5 & 43.5 & 45.5 & 47.5 & 51 & 54.5 & 59.5 \\ T_{c} & 0 & 63 & 146 & 209 & 261 & 355 & 512 & 523 & 564 & 585 & 606 & 627 & 658 & 690 & 732\end{array}$

Table 1: Combinations of $\Delta T$ and $T_{c}$ (both in msec) for $t_{0}=209.4 \mathrm{msec}$, obtained by least-squares fits with a variance within $0.025 \%$ of the best fit. Obviously the quality of the fit hardly depends on the pairs of values listed.

\section{Ideas for Future Work}

Equation (5) predicts that, if $t_{0}$ is supposed to remain fixed, an increase of $\langle T\rangle$ implies a decrease of $\Delta T$ (and vice versa). This prediction is at variance with another, hierarchically conceived proposal regarding $\langle T\rangle$ as integrating a particular number of elementary update intervals so that $\langle T\rangle$ is a multiple of $\Delta T$ [32]. Since $\langle T\rangle$ shows considerable interindividual variations, it is in principle possible to distinguish between the two approaches on an empirical basis: Measuring $\Delta T$ as a function of $\langle T\rangle$ for subjects with sufficiently different $\langle T\rangle$ should yield accordingly different values of $\Delta T$.

Much empirical material relevant in this context was collected in [42], with a special emphasis on clinical implications of temporal perception on the time scales mentioned above. Unfortunately, they did not report covariations of different time scales for the same individuals, so Eq. (5) cannot be tested using their published data.

Among other results, it was found [42] that $\Delta T$ is significantly increased in patients with posterior left-hemispheric lesions with fluent aphasic syndromes. In these patients, and in children suffering from language-learning impairments or dyslexia, behaviorally-oriented training was shown to reduce $\Delta T$ down to the normal range so that the ability to resolve rapidly presented stimuli was (partly) regained.

It is plausible that the size of the sequential order threshold $\Delta T$ can be regarded as an elementary update interval characterizing the temporal resolution of perceptual awareness. This resolution can possibly be further increased by particular techniques among which meditative practice may play a role. In the frequency domain, specific features of $\gamma$-activity have been reported $[43,44]$ in particular meditative states and interpreted these results in terms of different modes of awareness.

In the time domain, recent observations [45] on experienced meditators are eminently interesting since the reported increase of $\langle T\rangle$ exceeds two orders of magnitude during specific kinds of meditation. This has to be compared with a much smaller factor of up to five for interindividual variations as they are commonly known. For meditators, it should therefore be possible to detect the relationship between $\langle T\rangle$ and $\Delta T$ with evidentiary significance if both time scales are co-determined.

According to the Necker-Zeno model (Eq. (5)), an enormously increased mean dwell time $\langle T\rangle$ is accompanied either by a corresponding decrease of $\Delta T$ or by an increase of $t_{0}$ (or by a combination of both). This would imply either an extraordinary enhancement of the time resolution of perceptual awareness, based on a very 
rapid update of processed information, or a drastically delayed decay of the cognitive state in reaction to a perceived stimulus. The relative impact of the time scales $\Delta T$ and $t_{0}$ can be tested with existing experimental designs, combining measurements of sequential order thresholds, of decay times of the cognitive state, and of dwell times of bistable stimuli. In addition, it would be interesting to study how discontinuous stimulus presentation modulates dwell times during meditation.

\section{Summary}

A quantitative model called Necker-Zeno model, developed in [21] for the description of the reversal dynamics in the perception of bistable stimuli, has been refined and extended to fit additional empirical results not considered so far. In particular, we focused (i) on the distribution $P(T)$ of dwell times $T$ or, respectively, reversal rates and (ii) on the behavior of mean dwell times $\langle T\rangle$ under discontinuous presentation of the stimulus. It turned out that the Necker-Zeno model is in good agreement with the analyzed experimental data.

With respect to the distribution $P(T)$, we refined the model by accounting for an initial (transient) phase of the dynamics which is highly plausible. This can be achieved in two formally different ways that lead to different forms of $P(T)$. Recent results [24] suggest a criterion to distinguish one of them as more appropriate on an empirical basis. We may speculate that some kind of attention relaxation is a significant factor for a cognitive interpretation of the competing kinds of initial behavior.

With respect to the mean dwell times $\langle T\rangle$ under discontinuous stimulus presentation, we extended the model by accounting for short presentation off-times. In addition to the correct prediction of an increasing $\langle T\rangle$ for increasingly long off-times reported earlier [21], the Necker-Zeno model does also correctly describe an increase of $\langle T\rangle$ for decreasingly short off-times recently reported in [27]. These results are non-trivial since they represent opposing trends for long and short off-times, separated by a critical time scale of the order of $300 \mathrm{msec}$.

Finally, we indicate some ideas and options for further empirical tests of the Necker-Zeno model. They are essentially based on the fact that the model predicts a fairly simple quantitative relation (Eq. (5)) between three significant cognitive time scales that are often discussed to be of the order of approximately 30, 300, and 3000 milliseconds. Recent observations [45] suggest that they can be dramatically changed under specific conditions.

\section{Acknowledgments}

We are grateful to four anonymous referees for their comments and suggestions how to improve an earlier version of this paper. 


\section{References}

[1] P. Kruse and M. Stadler, Eds., Ambiguity in Mind and Nature. Berlin: Springer, 1995.

[2] F. Tong, "Competing theories of binocular rivalry: A possible solution", Brain and Mind, vol. 2, pp. 55-83, 2001.

[3] R. Blake and N. Logothetis, "Visual competition", Nature Neuroscience, vol. 3, pp. 1-11, 2002.

[4] G.M. Long and T.C. Toppino, "Enduring interest in perceptual ambiguity: Alternating views of reversible figures", Psychological Bulletin vol. 130, pp. 748$768,2004$.

[5] A. Borsellino, A. de Marco, A. Alazetta, S. Rinesi, and R. Bartolini, "Reversal time distribution in the perception of visual ambiguous stimuli", Kybernetik, vol. 10, pp. 139-144, 1972.

[6] S.R. Lehky, "Binocular rivalry is not chaotic", Proceedings of the Royal Society London B, vol. 259, pp. 71-76, 1995.

[7] M. Aafjes, J.E. Hueting, and P. Visser, "Individual and interindividual differences in binocular retinal rivalry in man", Psychophysiology, vol. 3, pp. 18-22, 1966.

[8] C.H. Ammons and R.B. Ammons, "Perspective reversal as affected by physical characteristics of Necker cube drawings", Proc. Mont. Acad. Soc., vol. 23, pp. 287-302, 1963.

[9] P. Walker, "Binocular rivalry: central or peripheral selective processes?" Psychological Bulletin, vol. 83, pp. 376-389, 1978.

[10] R. van Ee, L.C.J. van Dam, and G.J. Brouwer, "Voluntary control and the dynamics of perceptual bi-stability", Vision Research, vol. 45, pp. 41-55, 2005.

[11] R. van Ee, "Dynamics of perceptual bi-stability for stereoscopic slant rivalry and a comparison with grating, house-face, and Necker cube rivalry", Vision Research, vol. 45, pp. 29-40, 2005.

[12] M. Meng and F. Tong, "Can attention selectively bias bistable perception? Differences between binocular rivalry and ambiguous figures", Journal of Vision, vol. 4, pp. 539-551, 2004.

[13] D. Alais and R. Blake, Binocular Rivalry. Cambridge: MIT Press, 2005.

[14] C. Basar-Eroglu, D. Strüber, M. Stadler, and E. Kruse, "Multistable visual perception induces a slow positive EEG wave", International Journal of Neuroscience, vol. 73, pp. 139-151, 1993. 
[15] J. Kornmeier and M. Bach, "Early neural activity in Necker cube reversal: Evidence for low-level processing of a gestalt phenomenon", Psychophysiology, vol. 41, pp. 1-8, 2004.

[16] D. Strüber, C. Basar-Eroglu, E. Hoff, and M. Stadler, "Reversal-rate dependent differences in the EEG gamma-band during multistable visual perception", International Journal of Psychophysiology, vol. 38(3), pp. 243-252, 2000.

[17] U. Isoglu-Alkac, C. Basar-Eroglu, A. Ademoglu, T. Demiralp, M. Miener, and M. Stadler, "Analysis of the electroencephalographic activity during Necker cube reversals by means of the wavelet transform", Biological Cybernetics, vol. 79, pp. 437-442, 2000.

[18] H.R. Wilson, "Computational evidence for a rivalry hierarchy in vision", Proceedings of the National Academy of Sciences of the US, vol. 100, pp. 1449914503, 2003.

[19] A.W. Freeman, "Multistage model for binocular rivalry", Journal of Neurophysiology, vol. 94, pp. 4412-4420, 2005.

[20] C.R. Laing and C.C. Chow, "A spiking model for binocular rivalry", J. Comput. Neuroscience, vol. 12, pp. 39-53, 2002.

[21] H. Atmanspacher, T. Filk, and H. Römer, "Quantum Zeno features of bistable perception", Biological Cybernetics, vol. 90, pp. 33-40, 2004.

[22] N. Fürstenau, "Modelling and simulation of spontaneous perceptioin switchingwith ambiguous visual stimuli in augmented vision systems", in Lecture Notes in Computer Science 4021. Berlin: Springer, 2006, pp. 20-31.

[23] P. Mamassian and R. Goutcher, "Temporal dynamics in bistable perception", Journal of Vision, vol. 5, pp. 361-375, 2005.

[24] J.W. Brascamp, R. van Ee, W.R. Pestman, and A.V. van den Berg, "Distributions of alternation rates in various forms of bistable perception", Journal of Vision, vol. 5, pp. 287-298, 2005.

[25] G.J. Kalarickal, and J.A. Marshall, "Neural model of temporal and stochastic properties of binocular rivalry", Neurocomputing, vol. 32-33, pp. 843-853, 2000.

[26] J. Kornmeier, Wahrnehmungswechsel bei mehrdeutigen Bildern-EEG-Messungen zum Zeitverlauf neuronaler Mechanismen. PhD thesis, University of Freiburg, 2002.

[27] J. Kornmeier, W. Ehm, H. Bigalke, and M. Bach, "Discontinuous presentation of ambiguous figures: How interstimulus-interval durations affect reversal dynamics and ERPs", Psychophysiology, vol. 44, pp. 552-560, 2007. 
[28] B. Misra and E.C.G. Sudarshan, "The Zeno's paradox in quantum theory", Journal of Mathematical Physics, vol. 18, pp. 756-763, 1977.

[29] H. Atmanspacher, H. Römer, and H. Walach, "Weak quantum theory: Complementarity and entanglement in physics and beyond", Foundations of Physics, vol. 32, pp. 379-406, 2002.

[30] E. Ruhnau, "Time Gestalt and the observer", in Conscious Experience, ed. by T. Metzinger. Exeter: Imprint Academic, 1995, pp. 165-184.

[31] H.P. Stapp, "Attention, intention, and will in quantum physics", Journal of Consciousness Studies, vol. 6, pp. 143-164 (particularly p. 159), 1999.

[32] E. Pöppel, "A hierarchical model of temporal perception", Trends in Cognitive Science, vol. 1, pp. 56-61, 1997.

[33] S. Dornic, "Measurement of satiation in reversible figures", Studia Psychologica, vol. 9, pp. 18-24, 1967.

[34] J.R. Price, "Effect of extended observation on reversible perspective duration", Psychonomic Science, vol. 16, pp. 75-76, 1969.

[35] D. Reisberg and M. O'Shaughnessy, "Diverting subjects' concentration slows figural reversals", Perception, vol. 13, pp. 461-468, 1984.

[36] D. Vickers, "A cyclic decision model of perceptual alternation", Perception, vol. 1, pp. 31-48, 1972.

[37] J. Orbach, D. Ehrlich, and H. Heath, "Reversibility of the Necker cube: I. An examination of the concept of 'satiation of orientation'", Percept. Mot. Skills, vol. 17, pp. 439-458, 1963.

[38] J. Orbach, E. Zucker, and R. Olson, "Reversibility of the Necker cube: VII. Reversal rate as a function of figure-on and figure-off durations", Percept. Mot. Skills, vol. 22, pp. 615-618, 1966.

[39] D.A. Leopold, M. Wilke, A. Maier, and N. Logothetis, "Stable perception of visually ambiguous patterns", Nature Neuroscience, vol. 5, pp. 605-609, 2002.

[40] A. Maier, M. Wilke, N. Logothetis, and D.A. Leopold, "Perception of temporally interleaved ambiguous patterns", Current Biology, vol. 13, pp. 1076-1085, 2003.

[41] J.K. Grossmann and A.C. Dobbins, "Competition in bistable vision is attributespecific", Vision Research, vol. 46, pp. 285-292 (2006). 
[42] N. von Steinbüchel, M. Wittmann, and E. Szelag, "Temporal constraints of perceiving, generating, and integrating information: Clinical indications", Restorative Neurology and Neuroscience, vol. 14, pp. 167-182, 1999.

[43] D. Lehmann, P.L. Faber, P. Achermann, D. Jeanmonod, L.R.R. Gianotti, and D. Pizzagalli, "Brain sources of EEG gamma frequency during volitionally meditation-induced, altered states of consciousness, and experience of the self", Psychiatry Research, vol. 108, pp. 111-121, 2001.

[44] A. Lutz, L.L. Greischar, N.B. Rawlings, M. Ricard, and R.J. Davidson, "Longterm meditators self-induce high-amplitude gamma synchrony during mental practice", Proc. Natl. Acad. Sci. USA, vol. 101, pp. 16369-16373, 2004.

[45] O.L. Carter, D.E. Presti, C. Callistemon, Y. Ungerer, G.B. Liu, and J.D. Pettigrew, "Meditation alters perceptual rivalry in Tibetan Buddhist monks", Current Biology, vol. 15, pp. R412-R413, 2005.

(C) Atmanspacher et al.; Licensee Bentham Open.

This is an open access article distributed under the terms of the Creative

Commons Attribution License (http://creativecommons.org/licenses/by/2.5/),

which permits unrestrictive use, distribution, and reproduction in any medium,

provided the original work is properly cited. 